\title{
DELIMITAC̣ÃO DOS NÚCLEOS TALÂMICOS PELA ELETROFISIOLOGIA ESTEREOTÁXICA
}

\author{
Nilton Luís LatuF * \\ Marcus J. Colbachini ** \\ José A. Braz Galvão **
}

Com o advento do L-Dopa e da Amentadina, a cirurgia estereotáxica vem sendo cada vez menos indicada no tratamento do parkinsonismo. Entretanto, nossa experiência $€ \mathrm{~m} 91$ casos de parkinsonismo ${ }^{8}$, assim como a de vários autores ${ }^{6}$, mostra que ainda existem indicações para a cirurgia nos casos de tremores unilaterais sem rigidez e nos casos cujo tratamento clínico não tenha determinado resultados satisfatorios quanto ao tremor.

Neste tipo de cirurg:a a dificuldade maior diz respeito à exatidão com que deve ser atingido o alvo visado. Este alvo é delimitado, pela maior parte dos cirurgiões apenas pela radiologia, com o risco de complicações mais ou menos graves que podem ser evitadas, conforme mostram Guiot e col., mediante a delimitação exata do alvo visado. Em uma secção sagital (Fig. 1), a zona de destruição eficaz tem a forma de um triângulo limitado, posteriormente, pelo núcleo ventral posterior (V.P.), inferiormente pelo corpo de Luys (L) e, anteriormente, pela cápsula interna. Em secção horizontal (Fig. 2) o alvo é limitado pela cápsula interna, pelo núcleo ventral posterior e, internamente, por uma linha que segue o plano da lâmina medular interna, com o feixe de Vicq'Azyr (AZ) e o pedúnculo anterior do tálamo, cujas lesões levam à confusão mental e afonia, respectivamente. Enfim, $\in \mathbf{m}$ secção frontal (Fig. 3) com a forma de uma cornucópia, encontra-se internamente o núcleo dorsal mediano (DM) cuja lesão provoca distúrbios mentais, lateralmente a cápsula interna $e$, inferiormente, a zona incerta ( $\mathrm{Zi}$ ) e o campo de Forel.

Vários foram os recursos empregados na talamotomia estereotáxica afim de serem determinados com exatidão os núcleos talâmicos ventrais (estimulação elétrica, refrigeração localizada ${ }^{2}$, registro das atividades elétricas celulares ${ }^{5}$ ).

\section{MATERIAL E METODOS}

O registro das atividades elétricas celulares, com pesquisa de potenciais evocados, proposto por Guiot e col. ${ }^{5}$ foi empregado por nós em 23 talamotomias. O método mostrou ser útil quanto à somatotopia da representação táctil no núcleo ventral posterior e quanto à delimitação tálamo-capsular, demonstrada pelos resultados obtidos.

Trabalho do Serviço de Neurocirurgia da Santa Casa de Ribeirão Preto, apresentado no IX Congresso da Sociedade Brasileira de Neurocirurgia (Rio de Janeiro, 15-20 julho, 1972): * Neurocírurgião-chefe; ** Residentes. 


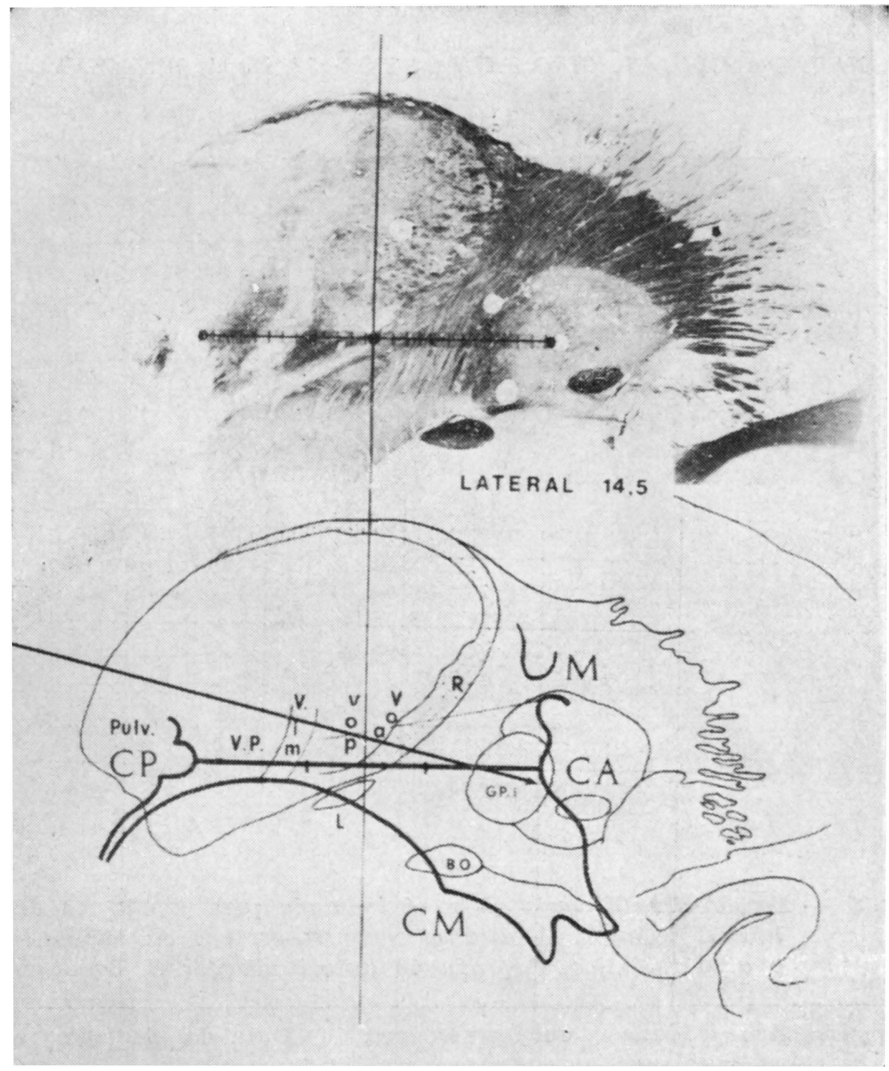

Fig. 1 - Secção sagital à $14,5 \mathrm{~mm}$ de lateralidade cruzando o alvo talamico. A flecha indica a trajetória utilizada. Reprodução autorizada por G. Guiot e col. ${ }^{5}$.

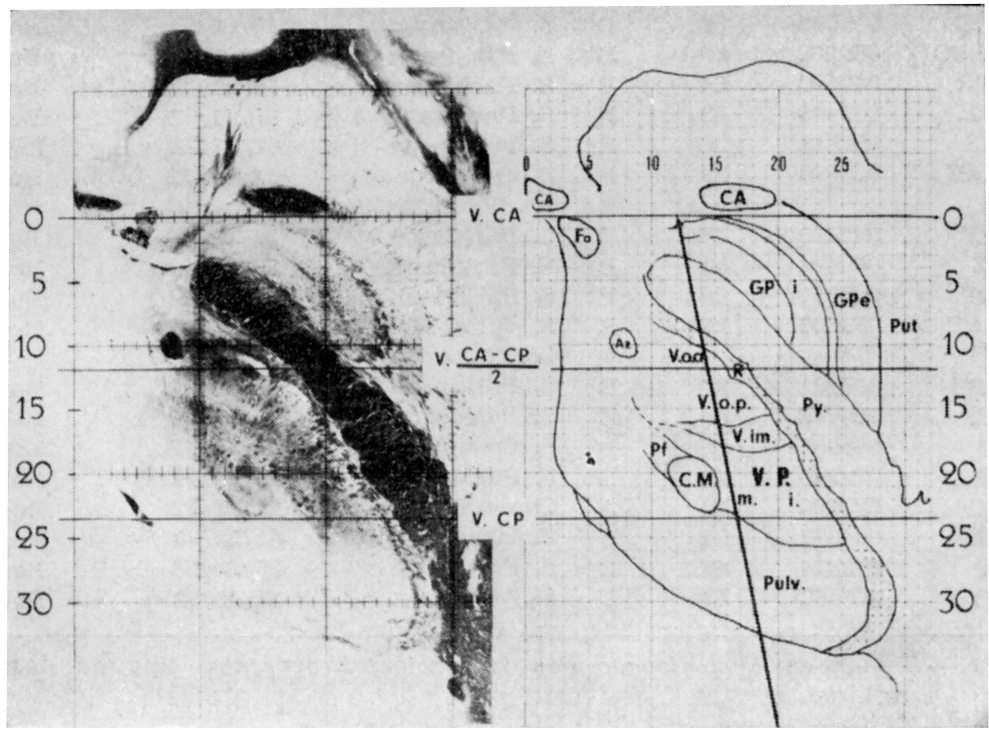

Fig. 2 - Seccão horizontal no plano da trajetória verificada na figura 1. Reprodução autorizada por G. Guiot e col. ${ }^{5}$. 


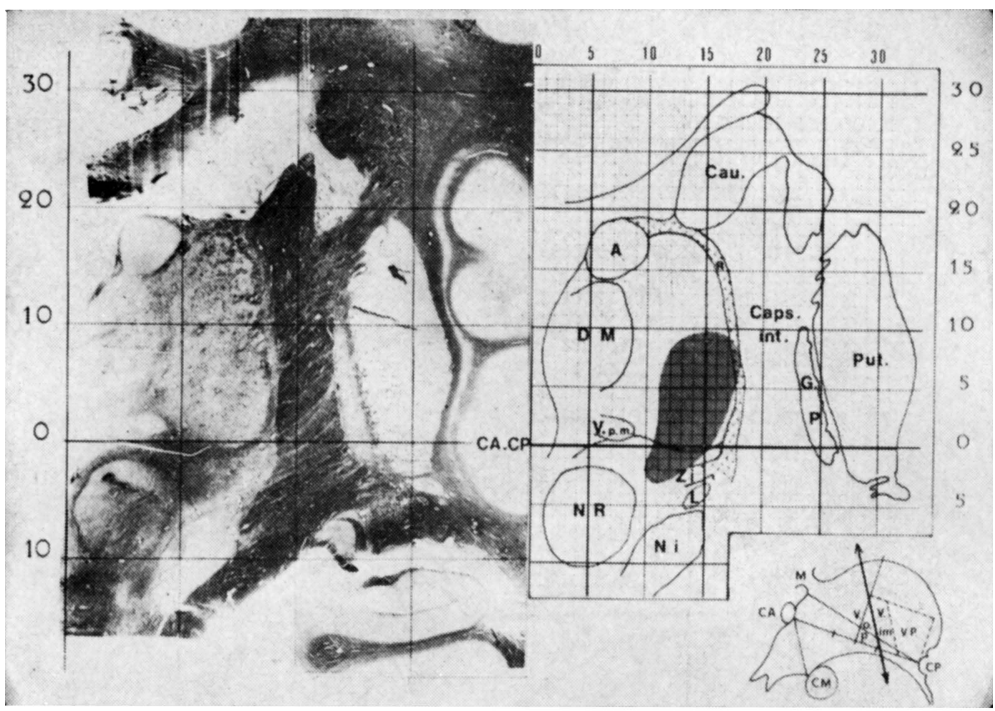

Fig. 3 - Seccão frontal cujo plano é indicado pela flecha no desenho lateral inferior. $\grave{A}$ direita encontra-se, em coloração escura, o alvo talâmico. Reprodução autorizada por G. Guiot e col. 5 .

\begin{tabular}{|c|c|c|c|c|c|c|}
\hline Casos & Registro & Idade & Cor & Doença & a da cirurgia & Resultado \\
\hline $1-\mathrm{ESA}$ & 041042 & 57 & B & S. Parkinson & $10 / 02 / 71$ & Regular \\
\hline $2-\mathrm{MM}$ & 040554 & 64 & B & S. Parkinson & $30 / 03 / 71$ & Bom \\
\hline $\mathbf{3}-\mathbf{A G}$ & 048836 & 69 & B & S. Parkinson & $07 / 05 / 71$ & Bom \\
\hline 4 - MGR & 041399 & 52 & B & S. Parkinson & $20 / 05 / 71$ & Bom \\
\hline 5 - OPS & 048111 & 54 & B & S. Parkinson & $04 / 06 / 71$ & Bom \\
\hline $6-\mathrm{LCC}$ & 051556 & 52 & B & S. Parkinson & $18 / 06 / 71$ & Bom \\
\hline 7 - RRSO & 052202 & 59 & B & S. Parkinson & $12 / 07 / 71$ & Bom \\
\hline $8-\mathrm{JTR}$ & 049853 & 64 & B & S. Parkinson & $11 / 11 / 71$ & Bom \\
\hline $9-J S F$ & 042409 & 48 & B & S. Parkinson & $10 / 11 / 71$ & Bom \\
\hline 10 - JJS & 057381 & 56 & B & S. Parkinson & $07 / 12 / 71$ & Regular \\
\hline 11 - MAF & 053803 & 19 & B & $\begin{array}{l}\text { S. Gilles de } \\
\text { La Tourette }\end{array}$ & $14 / 01 / 72$ & Bom \\
\hline $12-J S F$ & 042409 & 48 & B & S. Parkinson & $31 / 01 / 72$ & Bom \\
\hline $13-\mathrm{AZ}$ & 062404 & 51 & B & S. Parkinson & $06 / 04 / 72$ & Regular \\
\hline $14-\mathrm{TM}$ & 065550 & 46 & $\mathbf{P}$ & S. Parkinson & $27 / 06 / 72$ & Bom \\
\hline $15-\mathrm{MAF}$ & 053803 & 19 & B & $\begin{array}{l}\text { S. Gilles de } \\
\text { La Tourette }\end{array}$ & $23 / 08 / 72$ & Bom \\
\hline 16 - MBS & 069191 & 37 & B & S. Parkinson & $20 / 09 / 72$ & Bom \\
\hline $17-\mathrm{CSF}$ & 069788 & 56 & B & S. Parkinson & $21 / 09 / 72$ & Bom \\
\hline $18-\mathrm{JC}$ & 068995 & 65 & B & S. Parkinson & $27 / 09 / 72$ & Bom \\
\hline 19 - JRSF & 072239 & 49 & B & S. Parkinson & $23 / 11 / 72$ & Bom \\
\hline $20-\mathrm{MO}$ & 072690 & 19 & A & Coreo-ateotose & $19 / 12 / 72$ & Regular \\
\hline $21-\mathrm{MM}$ & 074577 & 24 & B & Espasmo de torção & $01 / 02 / 73$ & Regular \\
\hline $22-\mathrm{AP}$ & 078314 & 46 & B & S. Parkinson & $30 / 04 / 73$ & Bom \\
\hline $23-\mathrm{LC}$ & 078966 & 53 & B & S. Parkinson & $11 / 05 / 73$ & Bom \\
\hline
\end{tabular}

Quadro 1 - Dados de identificação dos 23 pacientes operados, tipo de doença $e$ resultados. 
Antes do ato cirúrgico foi feita uma pneumencefalografia para estabelecer as coordenadas radiologicas das estruturas visadas; as coordenadas de altura e de profundidade são obtidas pelo clichê de perfil e a de lateralidade pelo cliché ânteroposterior. Com os reparos tomados nestas chapas construímos a projeção lateral dos núcleos ventrais cujas medidas não nos dão garantia absoluta não só por ter o terceiro ventrículo a forma de um disco bicôncavo, como também pela presença ou ausência da comissura branca influenciando na lateralidade 4 e, portanto, na coordenada mais difícil de precisar-se, justificando o controle fisiológico.

O ato cirúrgico é realizado sob neuroleptanalgesia utilizando-se o aparelho e a técnica estereotáxica de Guiot, cuja sonda de penetraçáo leva no seu interior um eletrodo bipolar concêntrico conectado ao aparelho eletrencefalográfico, seguindo uma trajetória póstero-anterior obliqua em direção à linha de base (Fig. 4), controlada

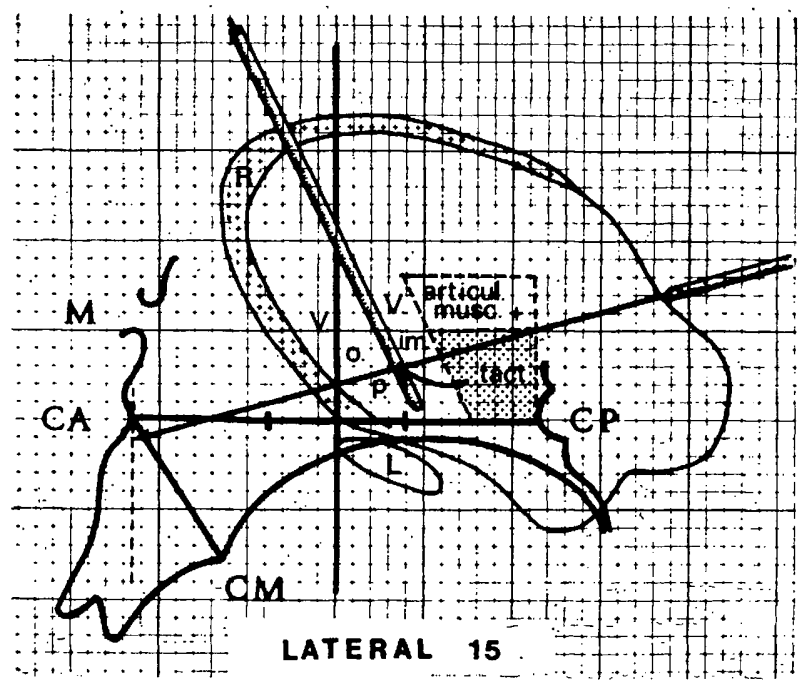

\footnotetext{
Fig. 4 - A flecha indica a trajetória, encontrando-se a zona táctil (V.P.) $5 \mathrm{~mm}$ acima de CA-CP, onde é feita a pesquisa neurofisiológica. Reprodução autorizada por Guiot e col. ${ }^{5}$.
}

pela iodoventriculografia praticada durante a intervenção cirúrgica. Esta trajetórla atravessa todos os núcleos talâmicos apresentando, portanto, vantagens sobre os outros métodos, pois várias estruturas podem ser destruidas com a mesma trajetória.

Cada núcleo pode ser reconhecido pelo traçado particular (Fig. 5) desde que se tenha tido inicialmente um pré-contacto talâmico. O pulvinar é caracterizado pelo ritmo alfa e pelo aparecimento de fusos de 7 à $12 \mathrm{c} / \mathrm{s} ;$ o núcleo ventral posterior (V.P.) caracteriza-se pela extrema densidade de "spikes" e pelos potencials evocados especificos tácteis, fornecendo as indicações topográficas precisas à uma somatotopia bem definida (Fig. 6).

A entrada no núcleo ventral intermediário (Vim) é reconhecida pelo desaparecimento dos potenciais evocados, precedidos de sua inversão. Esta inversão produz-se desde que a ponta do eletrodo penetra o núcleo Vim, enquanto que $o$ anel está ainda no núcleo VP. Ela é confirmada pelo aspecto dos spikes que são menos densos mas de grande amplitude e pelo aparecimento de fusos de ondas lentas com ritmo de 


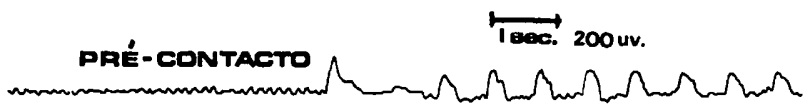

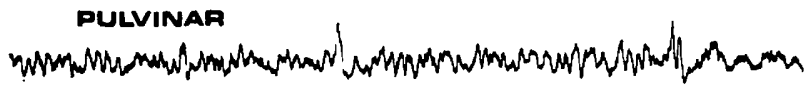

Dulvinas

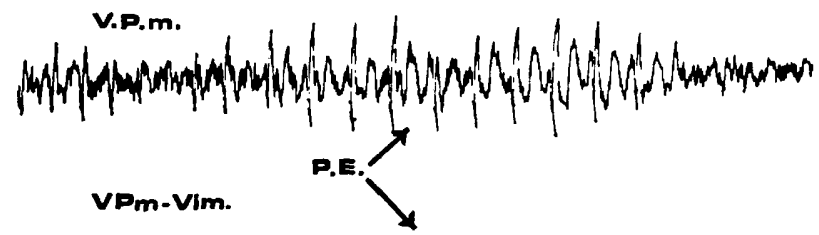

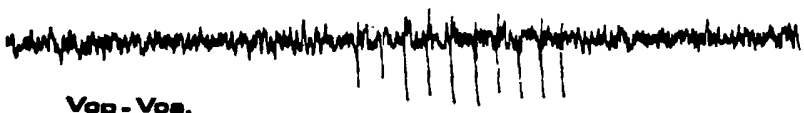

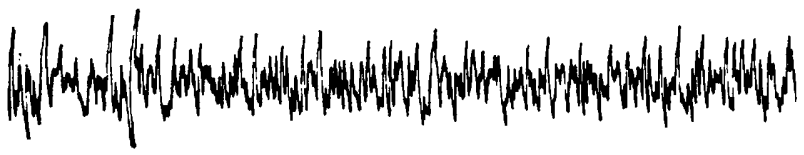

petrculapis-capgula.

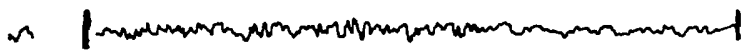

Fig. 5 - Traçado EEG do paciente T.M. Reg. 065550 obtido dos núcleos talamicos atravessados durante a cirurgia estereotáxica: pré-contacto (pulsações transmitidas próximas ao tálamo); pulvinar (ritmo alfa e fusos de sono); V.P.m. potenciais evocados; V.P.m. e V.I.m. (inversão dos potenciais evocados); V.o.p. e V.o.a. (fusos característicos); cápsula interna (achatamento do tracado).

20 à $25 \mathrm{c} / \mathrm{s}$. No núcleo ventral oral posterior (V.o.p.) a atividade dos spikes diminui bruscamente e o ritmo dos fusos de ondas lentas cai para $18-22 \mathrm{c} / \mathrm{s}$; uma nova reducão da atívidade spikes é observada no núcleo reticular havendo queda do ritmo para $15-20 \mathrm{c} / \mathrm{s}$. Finalmente, penetrando-se na cápsula interna, o eletrodo não recolhe mais spikes. 


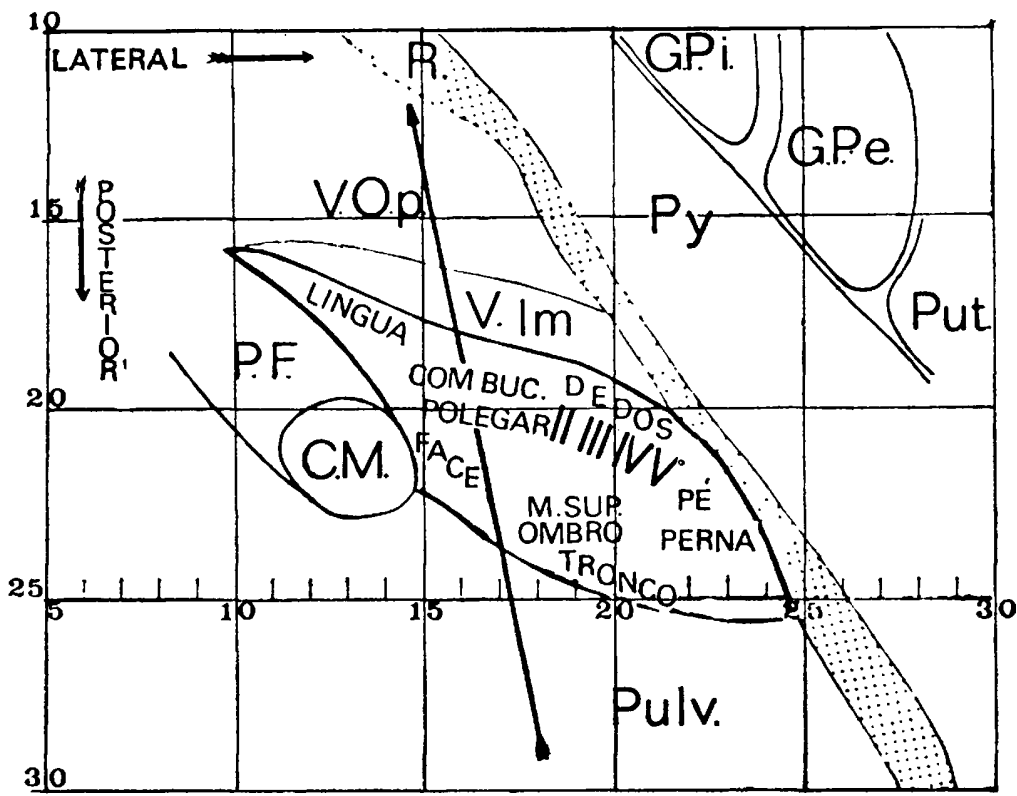

Fig. 6 - Secção horizontal mostrando a somatotopia da representação táctil do núcleo ventral posterior. A flecha indica a trajetória ideal para o tratamento cirúrgico dos tremores. Reprodução autorizada por G. Guiot e col. ${ }^{5}$.

A zona ideal do núcleo V.o.p., visada para o tratamento do tremor do membro superior é situada adiante da representacão táctil do polegar. Entretanto se ao penetrarmos no núcleo ventrolateral (Vim antes e Vop em seguida) o eletrodo registrar potenciais evocados nos últimos dedos da mão ou ainda no membro inferior, isso indica que a trajetória é muito lateral; ao inverso, quando os potenciais evocados são obtidos unicamente na face ou na língua, significa que a trajetória é muito interna, devendo ser corrigida.

Para o tremor do membro inferior a zona de destruição deve ocupar o canto póstero-lateral do alvo contra a cápsula interna, que se encontra adiante da representação táctil dos últimos dedos da mão. Para o tremor mandibular, a zona eficaz está adiante da representação táctil lahial.

\section{COMENTARIOS}

Como já demonstraram Guiot e col. ${ }^{4}$, o controle neurofisiológico reduz de mais ou menos $1 \mathrm{~mm}$ o êrro radiológico, afetando as coordenadas de lateralidade, altura e profundidade, o que é suficiente para determinar seqüelas pós-operatórias de acordo com as estruturas vizinhas atingidas. Assim sendo, mediante este método pode ser conseguida a delimitação exata da zona a ser destruída tendo como limite posterior o núcleo ventral posterior e, anteriormente, a cápsula interna; a lateralidade pode ser controlada mediante somatotopia, descrita por P. Derome ${ }^{3}$ e que é a mesma mostrada por Walker ${ }^{10}$ no núcleo ventral lateral, como também pela direção com a qual 
o eletrodo sai do tálamo para penetrar na cápsula interna (quanto mais externa mais precocemente a cápsula será encontrada). Os bons resultados obtidos nos levam a concordar com Guiot e col. ${ }^{4}$ que esta técnica permite corrigir alguns erros imputáveis às variações anatômicas individuais, permitindo identificar funcionalmente certos núcleos e delimitar melhor o núcleo ventral lateral cuja importância é reconhecida no tratamento cirúrgico dos movimentos involuntários.

\section{R E S U M O}

Os limites da área destruída durante a cirurgia estereotáxica são descritos levando em consideração as complicações decorrentes de lesões determinadas erroneamente. São comentados os métodos empregados com a finalidade de controlar a delimitação do alvo, sendo descrita a técnica usada em 23 talamotomias com derivação da atividade elétrica celular dos núcleos talâmicos atravessados e a pesquisa de potenciais evocados, graças à somatotopia da representação táctil no núcleo ventral posterior. Com este método reduz-se de mais ou menos $1 \mathrm{~mm}$ o êrro radiológico, prescisando-se o alvo terapêutico talâmico nos três planos de espaço.

\section{S U M M A R Y}

Delimitation of the thalamic nuclei by stereotaxic electrophysiology.

The limits of the area to be destroyed during the stereotaxic surgery for the treatment of tremors are described taking into account the complications due to lesions erroneously performed. The method applied is commented in order to control the accuracy of the target delimitation, describing the technique employed in 23 thalamotomies, recording the electrical activity of the thalamic nuclei acrossed and researching evoked potentials thanks to the somatotopic tactil representation in the ventral posterior nuclei. The method permits to reduce radiologic errors giving more accuracy for the delimitation of thalamic target in the three planes of space.

\section{REFER E N C I A S}

1. BERTRAND, C. \& JASPER, H. - L'enregistrement en micro-électrode d'unités thalamique an cours de la chirurgie estéréotaxique des mouvements involontaires. Neuro-Chirurgie 10:447, 1964.

2. COOPER, I. S. - Cryogenic surgery of the basal ganglia. J.A.M.A. 181:600, 1962.

3. DEROME, P. - Le noyau ventral posterieur chez l'homme. Thèse, Paris, 1965.

4. GUIOT, G.; ARFELL, G.; DEROME, P. \& IKAHN, A. - Procédés de controle neurophysiologique pour la thalamotomie estéréotaxique. Neuro-Chirurgie 14: $553,1968$. 
5. GUIOT, G.; DEROME, P. \& ARFEL, G. - Chirurgie stéréotaxique des syndromes extra-pyramidaux. Encyclopédie Médico-Chirurgicale: Neurologie 6:17.700 C10, 1968.

6. GUIOT, G.; HARDY, J. \& FESSARD, D. A. - Delimitation précise des structures sous corticales et identification des noyoux thalamiques chez l'homme par l'electrophysiologique stéréotaxique. Neuro-Chirurgie 5:1, 1962.

7. KRAYENBUHL, H. \& SIEGFRIED, J. - Traitement de la maladie de Parkinson: L-Dopa ou stéréotaxie?. Neuro-Chirurgie 16:71, 1970.

8. LATUF, N. L.; ColbachinI, M. J. \& GALvaO, J. A. B. - Tratamento do parkinsonismo com L-Dopa. Arq. de Neuro-Psiquiat (São Paulo) 30:138, 1972.

9. STELLAR, S.; MANDELL, S.; WALTZ, J. M. \& COOPER, I. S. - L-Dopa in the treatment of parkinsonism. J. Neurosurg. 32:275, 1970.

10. WALKER, A. E. - The Primate Thalamus. The University of Chicago Press, Chicago, 1938.

Serviço de Neurocirurgia - Santa Casa de Misericórdia - Caixa Postal 644 14100 Ribeirão Preto, SP - Brasil. 